\title{
FLOW MODULATION OF A PLANAR FREE SHEAR LAYER WITH LARGE BUBBLES-DIRECT NUMERICAL SIMULATIONS
}

\author{
M. TAEIBI-RAHNI, ${ }^{1}$ E. LOTH ${ }^{1}$ and G. TRYGGVASON ${ }^{2}$ \\ ${ }^{1}$ Department of Aeronautical and Astronautical Engineering, University of Illinois at Urbana-Champaign, \\ Urbana, IL 61801 and ${ }^{2}$ Department of Mechanical Engineering and Applied Mechanics, University of \\ Michigan, Ann Arbor, MI 48109, U.S.A.
}

(Received 12 October 1993; in revised form 21 March 1994)

\begin{abstract}
The flow of a planar free shear layer with cylindrical bubbles is simulated using a finite difference/front tracking scheme. This approach allows direct numerical simulation of the multiphase flow by wholly incorporating the local bubble flow field in conjunction with the large scale vortical structures of the liquid. The role of large bubbles in modifying low Reynolds number $(\sim 250)$ shear flow structures is investigated, specifically for bubbles whose diameter approaches the scale of the largest liquid eddies. The results indicate that duration of eddy crossing is the main mechanism for flow modulation, which is typically characterized by decreased vortex coherency and size, modified fluctuation statistics and significant variations in pairing/merging phenomena. The comparison of fluctuating statistics and flow field visualization also allowed qualitative discrimination between the modulation of the non-linear eddy dynamics and fluctuations due simply to the random bubble induced perturbations.
\end{abstract}

Key Words: free shear layer, two-phase flows, bubbles, modulation, direct numerical simulation

\section{INTRODUCTION}

In many bubbly flows, the fluid contains bubbles which cannot be simply treated as tracers that follow the convection of the surrounding flow field; instead, velocity non-equilibrium may lead to changes in both the bubble dispersion and the continuous phase fluctuation levels. Often, the nature of the interactions can be a two-way coupling where the dispersed phase trajectory is affected by the local unsteady turbulence of the continuous phase (turbulent dispersion) and where the displacement and trailing wake of the bubbles alter the turbulence of the liquid (turbulence modulation). This study concentrates on flow modulation, but at low Reynolds number such that the flow is not yet turbulent. Present two-phase flow turbulence modeling techniques require some empiricism and may not always provide robust predictive performance since the turbulent interphase transport mechanisms are still not fully characterized (Tishkoff 1989). It is thus helpful to understand the basic characteristics of bubbly flow modulation to improve interphase transport modeling and to support technology gains in related engineering systems.

\subsection{Previous experimental studies}

To investigate the role of the continuous phase structures in two-phase flows, attention must be first given to previous studies of the single-phase counterparts. Notable contributions have been derived from experiments with instantaneous observations. Brown \& Roshko (1974) and others have shown that large scale structures in single-phase planar free shear layers are still present, primarily two-dimensional, and coherent at high Reynolds numbers. The 2-D structures (termed eddies and braids) were found to represent the dominant entrainment mechanism for such flows and contain the bulk of the turbulent energy. In addition, the large scale structures in a single-phase planar free shear layer may also be actively excited by a driving frequency to yield increased coherency and growth rates for a significant convection distance, as shown by Ho \& Huang (1982). Thus, the free shear layer development is sensitive to disturbances under proper conditions.

The change of the fluctuations (or turbulence) due to the presence of the dispersed phase is referred to as turbulence modulation. Recently, Gore \& Crowe (1989) have surveyed measurements of turbulence modulation for a number of multiphase flows to identify the relationship between 
the continuous phase turbulence level and the dispersion length scale, $\beta$, defined as the ratio between dispersion size, e.g. bubble diameter $\left(d_{\mathrm{B}}\right)$ to the continuous phase integral length scale $(\Lambda)$. From these results, the authors concluded that for values of $\beta$ less than 0.07 , turbulence modulation results in a dampening of the continuous phase turbulent energy as compared to single phase flows; and for values of $\beta$ greater than 0.07 , turbulence modulation results in increased continuous phase turbulence, as much as three times the levels found in single-phase flows. One might reasonably expect that as $\beta$ approaches unity, the turbulent flow field might act differently than for an infinitesimally small $\beta$, due to the presence of strong gradients which have length scales on the order of the bubble size itself. However, the above trends of Gore \& Crowe were only valid for centerline turbulence values and broke down if overall turbulence was examined (Davis 1993). Also, Yuan \& Michaelides (1992) have theoretically noted that the energy dissipation due to the acceleration of the particle with respect to the continuous phase is the main cause of turbulence intensity reduction for most conditions, while the flow disturbance due to the motion of the particle and its wake is the main cause for turbulence enhancement. In addition, one must also be careful to distinguish true turbulence modulation (shear induced) which appears due to modification of continuous fluid eddy vorticity--from pseudo-turbulence (bubble induced)-which appears due to the random arrival of bubbles and their wakes at an Eulerian control volume (Lopez et al. 1992).

\subsection{Previous computation studies}

Several researchers have numerically simulated the single-phase free shear layer flow. Direct numerical simulations (DNS), large eddy simulation (LES) and Reynolds averaged turbulence modeling have been commonly used to describe the continuous phase. DNS of incompressible free shear layers were performed by Riley \& Metcalfe (1980), Metcalfe et al. (1987a, b), and Sandham \& Reynolds (1989). The above DNS studies, which involve no turbulence modeling, indicated that (a) use of appropriate initial disturbances was found to be essential in initiation of instabilities resulting in the free shear layer vortex rollups, (b) the developments of the mean flow were dominated by inviscid, two-dimensional Kelvin-Helmholtz instability effects and (c) growth was linked to vortex rollup and eddy amalgamation (primarily pairing).

In general, for numerical simulations of two-phase flow problems, mixed-fluid, separated-fluid and single-fluid physical models have been employed to take into account the dispersed phase (Faeth 1987). These different treatments of the dispersed phase refer to either neglection of velocity and temperature differences, use of a point mass treatment, or use of a finite size dispersed phase in the flow field, respectively. A simple mixed-fluid two-phase flow description is that of the locally homogeneous flow (LHF) approximations, where the flow field is effectively treated as single-phase flow with a variable density, written as a function of the mixture fraction, i.e. a passive scalar with no interphase momentum transport (Faeth 1987).

On the other hand, separated-fluid models allow relative velocity differences by treating the dispersed phase independently in either a Lagrangian or Eulerian format. Generally, this more complex but more accurate model, which treats the dispersed phase as a point mass, requires empirical correlations for the interphase transport mechanisms. To study a bubbly two-phase flow, a $k-\epsilon$ model was also used in the viscous separated-fluid model of Lopez et al. (1992). They compared their results with experimental data to verify that the linear superposition of a shear-induced and bubble-induced (pseudo-) turbulence may be reasonably used under some conditions.

Finally, single-fluid models allow direct description of the displacement and wake effects by simulating the flow around the dispersed phase. They may also include description of the flow field within the dispersed phase, e.g. within a bubble or drop, as well as description of the continuous phase flow, where a front tracking algorithm may be employed to prescribe the interphase boundary. These models are usually used when the length scale of the dispersed phase is significant compared with an integral length scale of the continuous phase because the interaction of the continuous phase can no longer be considered to act at a single point of the flow field. In the limits of either inviscid flow or Stokes flow, the problem can be reformulated in terms of an integral equation over the interface separating the two phases. This has been used by several investigators for both two-dimensional (Shankar 1992) and three-dimensional bubbles (Chahine \& Duraiswami 1992). Single-fluid bubble simulations using the full Navier-Stokes equations are less common, and 
include: axisymmetric computations of a single rising bubble by Ryskin \& Leal (1984) and three-dimensional computations of the collision of two bubbles by Unverdi \& Tryggvason (1992a, b). However, single-fluid DNS of large bubbles in a free shear layer have not been presented before to the authors' knowledge.

\subsection{Present study}

This study's main objective was to investigate the mechanisms which control modification of a low Reynolds number two-dimensional free shear layer, specifically, when the bubble sizes become significant compared to the size of the eddies in the surrounding fluid (this is only qualitatively related to turbulence modulation). The present flow field includes large bubbles with values of $\beta$ near unity for which modulation is expected to be strong. The study of such large bubbles may also be important to characterize influences of coherent bubble clouds, which can behave similar to a single large bubble. However, the most obvious application is for LES subgrid turbulence models where bubble diameters may be of the same order as the unresolved fluid scales, bubble deformation is small, and the subgrid Reynolds numbers are low.

To obtain a two-dimensional representation of the bubbly free shear layer flow, the computational effort included a time-accurate Navier-Stokes formulation of one or two bubbles embedded in a low Reynolds number free shear layer; to allow direct investigation of flow modulation. The simulations of this study employed a finite difference method which takes advantage of a front tracking scheme (based on Unverdi \& Tryggvason 1992a), that fully describes the flow within and outside the bubbles. These computations were completed using an unstructured adaptive grid on the bubble surface, in order to account for the bubble deformation.

At the low Reynolds numbers employed herein, the large-scale vortex rollup is still expected (Winant \& Browand 1974), but the small scale turbulent structure and three dimensionality associated with a fully-developed turbulent flow at higher Reynolds numbers $\left(\mathrm{Re}_{\delta}\right)$ are notably absent. In addition, the bubbles were limited to a two-dimensional shape. This approximation has been used recently to allow detailed information for rising bubbles (Vanden-Brock 1992) and for bubbly uniform flows (Shankar 1992, Zun et al. 1993). Also, Collins (1965) experimentally studied the structure and the behavior of the wakes behind two-dimensional air bubbles in water. The above two restrictions (low $\mathrm{Re}_{\delta}$ and 2-D flow) were severe but necessary based on available computational resources.

To study the character of the complex two-phase interactions, three situations were studied: a bubble rising in a quiescent liquid, a planar single-phase free shear layer flow and a bubbly free shear layer flow. The planar free shear layer was selected because its eddy structure is more coherent and accessible than other shear flows and has been widely documented as a single-phase flow, Inserting one or two large cylindrical bubbles in this flow permits clear interrogation of the effect on the eddies and braids, which are the dominant energy containing structures.

\section{NUMERICAL METHOD}

The DNS used in this study computes the unsteady, incompressible, viscous, immiscible, multi-fluid, two-dimensional Navier-Stokes equations for the liquid and the gas (viscous singlefluid model). The evolution of the free shear layer and the motion of the bubbles are governed by the Navier-Stokes equations, which in conservative form are:

$$
\frac{\mathrm{D}(\rho \mathbf{V})}{\mathrm{D} t}=-\nabla p+\rho \boldsymbol{g}+\nabla \cdot(2 \mu \mathbf{D})+\sigma \kappa \mathbf{n} \boldsymbol{\delta}\left(\mathbf{X}-\mathbf{X}^{\mathfrak{\gamma}}\right),
$$

where $\mathbf{D}$ is the rate of deformation tensor, with components: $D_{i j}=\left(V_{i, j}+V_{j, i}\right) / 2 ; \mathbf{V}$ is the velocity vector (liquid and the gas); $\sigma$ is the surface tension coefficient; $\kappa$ is the curvature; $\boldsymbol{g}$ is the gravity force; and $\mathbf{n}$ is a normal to the bubble surface. The density $(\rho)$ and viscosity $(\mu)$ are allowed to vary, such that these equations are therefore valid for the whole flow field (both the bubble and the ambient liquid flow fields), and surface tension forces have been added as a delta function, $\delta\left(\mathbf{X}-\mathbf{X}^{\mathfrak{f}}\right)$, which is non-zero only on the bubble surface, where $\mathbf{X}=\mathbf{X}^{\mathfrak{f}}$.

These equations are complemented by the incompressibility condition $(\nabla \cdot V=0)$, which leads to an elliptic pressure equation when combined with the Navier-Stokes equations, and the 
continuity equations for the density and viscosity:

$$
\frac{\partial \rho}{\partial t}+\mathbf{V} \cdot \nabla \rho=0, \quad \frac{\partial \mu}{\partial t}+\mathbf{V} \cdot \nabla \mu=0
$$

These equations simply state that the density and viscosity of a fluid particle does not change.

In order to capture the discontinuity of the flow between the gas and the liquid, an indicator function, $I(\mathrm{X})$, which is 0 for the outer liquid and 1 for the gas inside the bubble, is constructed using the known position of the points on the bubble surface. Therefore, the density and viscosity fields can be written in terms of $I(\mathbf{X})$ as follows:

$$
\rho(\mathbf{X})=\rho_{\mathrm{L}}+\left(\rho_{\mathrm{B}}-\rho_{\mathrm{L}}\right) I(\mathbf{X}) ; \quad \mu(\mathbf{X})=\mu_{\mathrm{L}}+\left(\mu_{\mathrm{B}}-\mu_{\mathrm{L}}\right) I(\mathbf{X}),
$$

where the interface function change is distributed over four computational cell lengths. The gradient of this function is referred to as the distribution function.

The Navier-Stokes equations are solved by a relatively standard finite difference projection method (Peyret \& Taylor 1986) on a staggered Cartesian grid. All spatial derivatives are evaluated by second order centered differences, and the time integration is performed by a second order predictor-corrector method. The pressure equation, which is non-separable since the density is not constant, is solved by a Black and Red SOR iteration technique. To advect the discontinuous density and viscosity fields, and to compute surface tension forces, we use a technique that is usually called the immersed boundary method and was introduced by Peskin (1977). This represents the bubble surface with separate computational elements, referred to as the front. The front grid is one-dimensional and is advected by the fluid velocity which is interpolated from the fluid grid. There is no numerical diffusion of this front since this thickness remains constant for all time, and from this front, the density and viscosity fields can be computed as described above. Therefore, the infinitely thin boundary is approximated by the smooth finite thickness distribution function (described above), that is also used to distribute the surface forces over the grid points close to the surface in a conservative manner. This technique, which is presented in more detail by Unverdi \& Tryggvason (1992a), has been used to simulate many flows including three-dimensional collision of two rising bubbles (Unverdi \& Tryggvason 1992b).

Since the flow inside the bubble typically contained much higher vorticity than the ambient liquid and since it is not the focus of this study, Favre-averaged quantities are calculated a posteriori to the simulation, to determine the outer fluid modulation. For example, the ambient liquid streamwise velocity is decomposed as:

$$
u(x, y, t)=\tilde{u}(y, t)+u^{\prime}(x, y, t)
$$

where, $\tilde{u} \equiv \overline{\rho u} / \bar{\rho}$ and $\overline{()}$ signifies average over a constant $y$ at a given time and where all velocity statistics were then averaged over the domain to provide total flow field Favre averages at a given time. These were then normalized by $\Delta u=u_{1}-u_{2}$, where subscripts 1 and 2 signify conditions at $y=+\infty$ and $y=-\infty$, respectively. Similarly, a Favre vorticity field was defined as: $\zeta \equiv \overline{\rho \omega} / \bar{\rho}$. Therefore, the flow dynamics within the bubble are given a significantly reduced emphasis, such that fluctuation statistics will be primarily indicative of the flow outside of the bubble.

\section{TEST CONDITIONS}

Test conditions for the flow simulations are described below and were chosen based on a modest variation of parameters and effects presumed to be important for flow modulation. For the rising bubble studies, bubble terminal Reynolds numbers, $\operatorname{Re}_{t}$ of about 65 and 133 (cases $A$ and $B$ ) were used, while computations of the single-phase and bubbly free shear layer flows (cases $\mathrm{C}-\mathrm{H}$ ) were limited to an initial liquid Reynolds number, $\operatorname{Re}_{\delta 0}\left(\equiv \rho_{\mathrm{L}} \Delta u \delta_{0} / \mu_{\mathrm{L}}\right)$ of about 250 .

The free shear layer development was temporal, based on periodic boundary conditions for the left and right boundaries and full slip conditions for the top and bottom walls (figure 1). As mentioned earlier, the flow evolution of any free shear layer flow is strongly sensitive to the initial perturbations. A fundamental perturbation $\left(w_{\mathrm{f}}\right)$ and a 90 degree out-of-phase subharmonic $\left(w_{\mathrm{s}}\right)$ superimposed on a hyperbolic tangent mean profile were employed based on the results of Metcalfe et al. (1987b) to yield the fastest vortex pairing. The initial velocity used in this study had the form: 


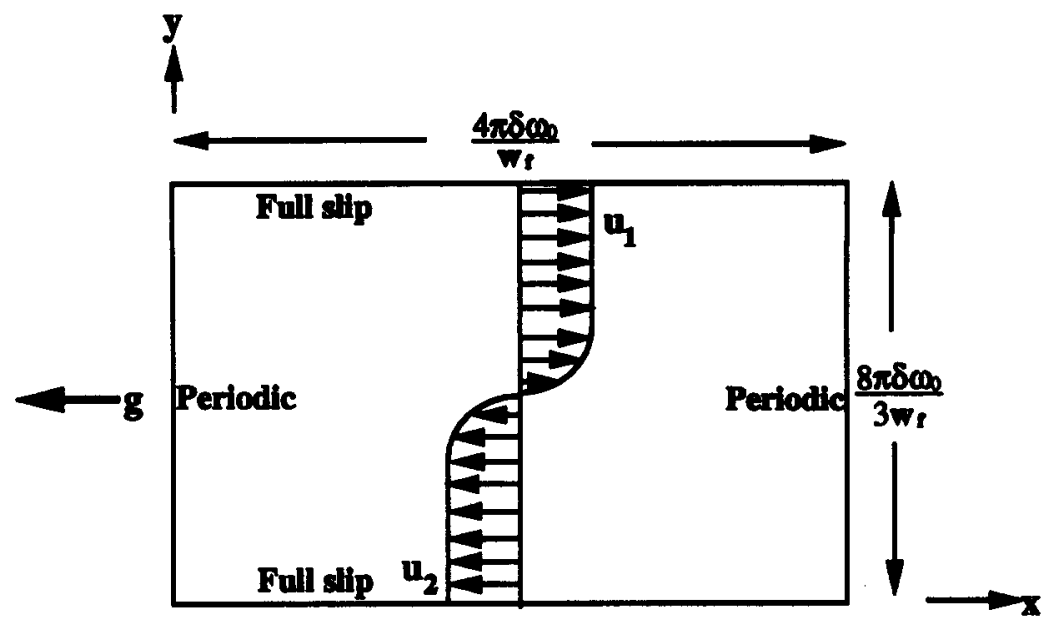

Figure 1. Computational domain with boundary and initial conditions.

$$
u(x, y, 0)=u_{1} \tanh \left(y / \delta_{\omega 0}\right)+2 u_{1} \operatorname{sech}\left(y / \delta_{\omega 0}\right)\left\{e_{\mathrm{f}} \cos \left[w_{\mathrm{f}}(x+\theta)\right]+e_{\mathrm{s}} \cos \left(w_{\mathrm{s}} x\right)\right\},
$$

where $e, w$ and $\theta$ are the amplitude, the wave number and the phase shift between the fundamental and subharmonic modes of the harmonic perturbation, respectively; and subscripts $s$ and $f$ signify the disturbances due to subharmonic and fundamental wave numbers. In this study, $u_{1}=2, e_{\mathrm{f}}=0.2$, $e_{\mathrm{s}}=0.14, w_{\mathrm{f}}=0.446, w_{\mathrm{s}}=w_{\mathrm{f}} / 2$ and $\theta=\pi / 2 w_{\mathrm{f}}$. Note in this description as in [1] all length scales were normalized by the initial vorticity thickness, $\delta_{00}\left(\equiv u_{1} /\left[\mathrm{d} u_{0} / \mathrm{d} y\right]_{\max }\right)$, and all velocity scales were normalized by $u_{1} / 2$. In order to yield two full fundamental waves, a flow domain of width $4 \pi / w_{\mathrm{f}}$ and height of $8 \pi / 3 w_{\mathrm{f}}$ is used (figure 1). Note, gravity (when added) will be in the streamwise direction indicating a vertical shear layer for which buoyancy would not force the bubble to escape the shear layer region of the flow.

To investigate the effects of the bubble on both the eddies and the braids of the free shear layer rollups, either one or two cylindrical bubbles were added to the single-phase free shear layer flow at $t=5$ : one in the vortex core (left bubble) and/or one on top of the connecting braid (right bubble). By this time, the free shear layer had already evolved and two large eddies and a middle braid had developed due to Kelvin-Helmholtz instability. This bubble addition was achieved by simply providing an instantaneous change to the density field while retaining the original velocity and pressure fields (except for surface tension), i.e. mass was instantly withdrawn at $t=5$, thus momentarily placing the bubble in instantaneous equilibrium. As will be noted in the results, no significant flow modulation follows this instantaneous bubble addition. Different effects of the bubbles' presence were studied by varying parameters for the five cases shown in table 1 . We first investigated the effect of only displacement (case D), then added the centripetal effect by changing the bubble density (case E) and finally added buoyancy by setting gravity to be non-zero (cases F-H), in order to differentiate these various effects. Note, a $\rho_{\mathrm{L}} / \rho_{\mathrm{B}}$ of 40 was used for the centripetal effect since higher ratios did not lead to significant changes in the flow but dramatically increased computational time. The theoretical bubble terminal velocity $\left(V_{t}\right.$, based on a fluid cylinder drag coefficient), Bond number $\left(E_{0} \equiv \rho_{\mathrm{L}} g d_{\mathrm{B}}^{2} / \sigma\right)$, Morton number $\left(\mathrm{M} \equiv g \mu_{\mathrm{L}}^{4} / \rho_{\mathrm{L}} \sigma^{3}\right)$ and Weber number (We $\equiv \rho_{\mathrm{L}} V_{\mathrm{t}}^{2} / \sigma$ ) are also listed in table 1 , along with the initial ratio of inviscid centripetal pressure gradients to buoyancy pressure gradients (defined as the eddy Froude number, $\mathrm{Fr}_{\delta}$ ) and the ratio of convection to buoyancy forces $(\xi)$, where

$$
\operatorname{Fr}_{\delta} \equiv \frac{\partial p}{\partial r} / \frac{\partial p}{\partial x} \sim\left[\frac{\frac{1}{2} \rho_{\mathrm{L}}(\Delta u / 2)^{2}}{\frac{\delta}{2}}\right] /\left[\rho_{\mathrm{L}} \boldsymbol{g}\right] \sim \frac{\Delta u^{2}}{4 \delta \boldsymbol{g}},
$$

and

$$
\xi=\left(\frac{\Delta u}{V_{1}}\right)^{2}
$$




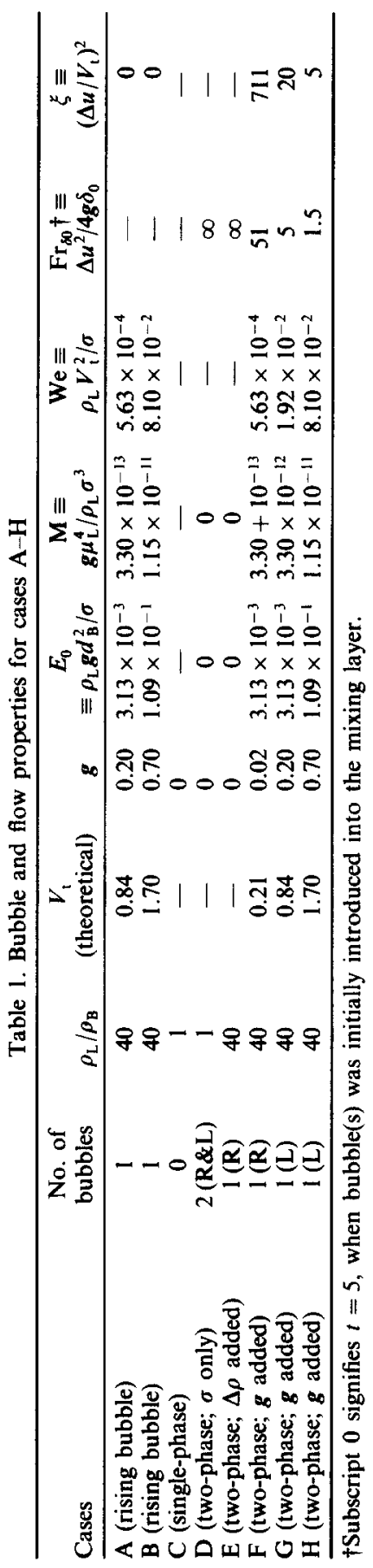


In this study, $E_{0}, \mathrm{M}$ and We were chosen such that the bubbles' deformation was minimal (in order to simplify the flow physics) and such that centripetal and buoyancy effects would be significant as compared to viscous advection as described above. Since the present flow is not fully turbulent, we will define $\beta$ herein as $d_{\mathrm{B}} / \delta$, where $\delta$ is the streamwise-averaged shear layer thickness based on the 5 and $95 \%$ levels of the mean velocity profile. All flows had an initial $\beta$ of 0.83 , which allowed clear observation of eddy modulation. The void fraction $(\epsilon)$ is based on the free shear layer domain (shear layer thickness times streamwise length of computational domain) and for one bubble was estimated to be about $9 \%$ upon introduction at $t=5$. As $\delta$ increases, both $\beta$ and $\epsilon$ will change accordingly.

In order to find an appropriate mesh size, separate resolution studies were performed for the single-phase free shear layer flow (case $\mathrm{C}$ ), the rise of a bubble in a quiescent liquid (cases $\mathrm{A}$ and B), and the combined bubbly free shear layer flows (cases D-H). Taeibi-Rahni et al. (1992) showed that a $\operatorname{Re}_{\text {cell }}\left(\equiv \rho_{\mathrm{L}} \Delta u \Delta x / \mu_{\mathrm{L}}\right)$ of about 37 was needed for an accurate grid independent solution, based on case $C$ with $\operatorname{Re}_{\delta 0}=332$. The average variance between the vorticity fields from $\operatorname{Re}_{\text {cell }}=37$ to $\operatorname{Re}_{\text {cell }}=24$ was about $6.5 \%$, and from $\operatorname{Re}_{\text {cell }}=24$ to $\operatorname{Re}_{\text {cell }}=19$ was about $1 \%$ of the maximum vorticity. Vorticity is a very sensitive indicator of grid resolution and was therefore used to highlight any changes due to varying the cell Reynolds number. For cases $A$ and $B$, with $\operatorname{Re}_{\text {cell }}$ based on the terminal velocity, a similar conclusion was drawn. However, a resolution study of the combined cases indicated that a finer grid $\left(\mathrm{Re}_{\text {cell }}\right.$ based on $u_{1}$ of about 24 ) was needed to fully resolve the flow (see figure 2). This resolution was also confirmed with convergence of flow statistics. Based on this new $\operatorname{Re}_{\text {cell }}$, a $\operatorname{Re}_{\delta 0}$ of 250 and a rectangular domain $(144 \times 96)$ was used for all the bubbly flow results.

\section{RESULTS AND DISCUSSION}

In order to understand the basic flow characteristics of the two-dimensional flow, a bubble rising in a quiescent liquid (cases $\mathbf{A}$ and $\mathrm{B}$ ) was first studied. The drag coefficient of the bubbles determined from these studies were shown to be in good agreement with empirical data for a uniform flow over a fluid cylinder. The basic results of the single-phase free shear layer results (case C) are presented followed by cases of a large bubble in the free shear layer (cases D-H). As was expected, for the surface tension specified, the bubbles maintained nearly cylindrical shape with only slight deformations for all cases presented here. Note, the modest deformations were not a limitation of the code, but were used to simplify the physics. The modulation is examined initially from Favre-averaged vorticity contours followed by flow field mean and RMS statistics.

\subsection{Shear layer modulation of the flow field}

As can be seen from figures 3-8, contours of Favre vorticity $(\zeta)$ serve well as markers of the vortex structures and strength. The contour increments shown in these figures represent $10 \%$ of the difference between the highest maximum and the lowest minimum found throughout all of the runs. Because of this consistency, direct comparison can be made of the vorticity levels between both different times and different cases. For the cases with bubbles, dark gray contours are shown along the maximum distribution function of the front, indicating a portion of the finite thickness associated with the variation of density, viscosity, and surface tension normal the bubble surface. The bubble interior is then illustrated with a lighter shade of gray such that its internal vorticity contours can be seen. Note that in this study time is non-dimensionalized by $2 \delta \omega_{0} / u_{1}$.

Figure 3 shows the evolution of $\zeta$ for the single-phase flow (case C). Discrete vortices are already formed at $t=5$, when the bubbles are added for the two-phase flow cases. By $t=10$, two eddies are fully formed. The size difference between these two eddies is due to the initial asymmetric disturbance. By $t=15$, eddy interactions, in which the right eddy is being pulled (stretched) by the left eddy, have begun and the two vortices have started to pair. This stretching actually results in the overall decrease in the shear layer thickness $(\delta)$ over time (figure 9). By $t=20$, vorticity in the left eddy is being reduced and stretched (and to a lesser extent in the right eddy). Finally, by $t=25$, pairing is beginning to take place as evidenced by the rapid increase in the size of the right eddy which has begun to absorb the left eddy. Also, the center portion is now being stretched somewhat 

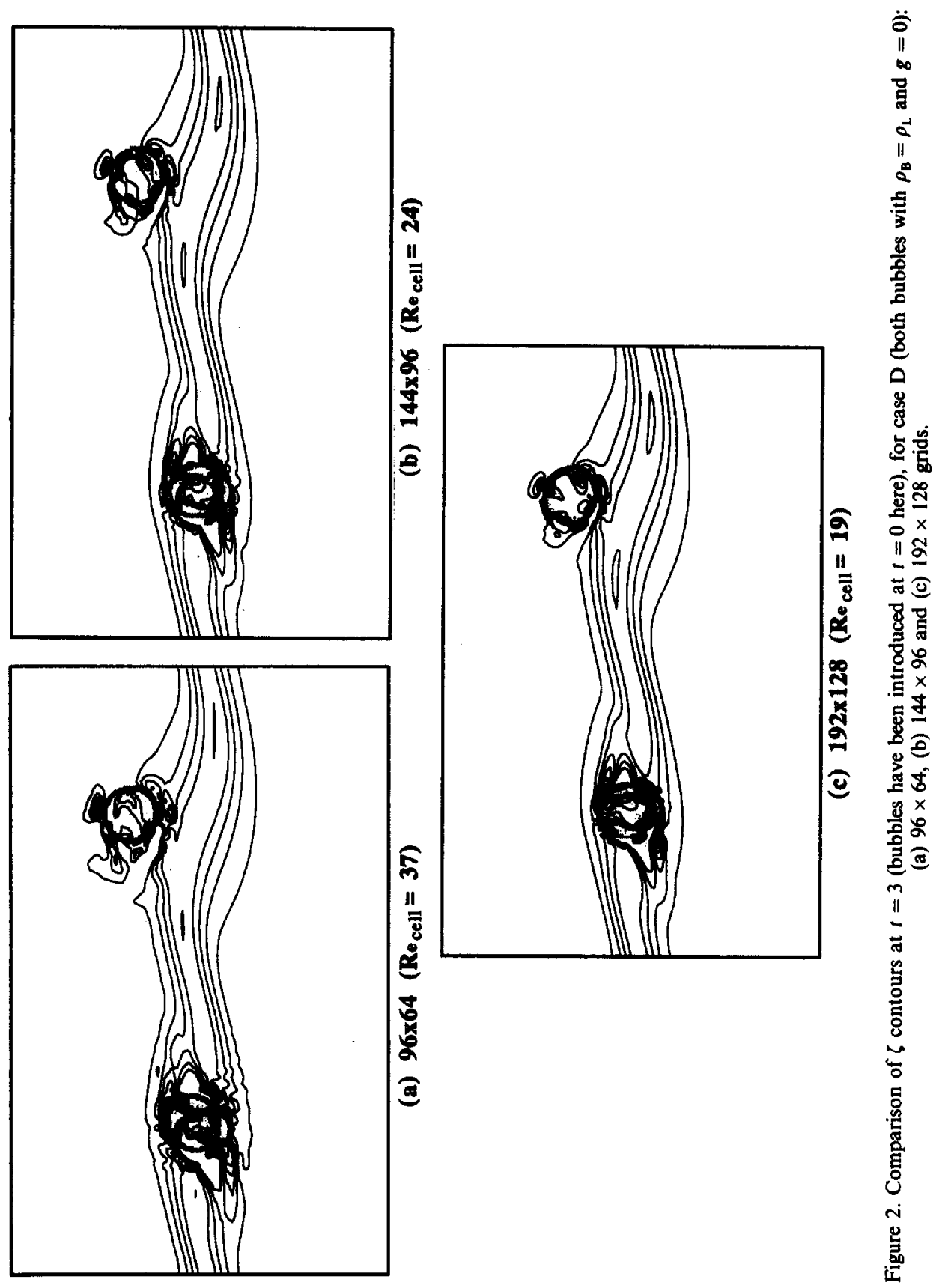


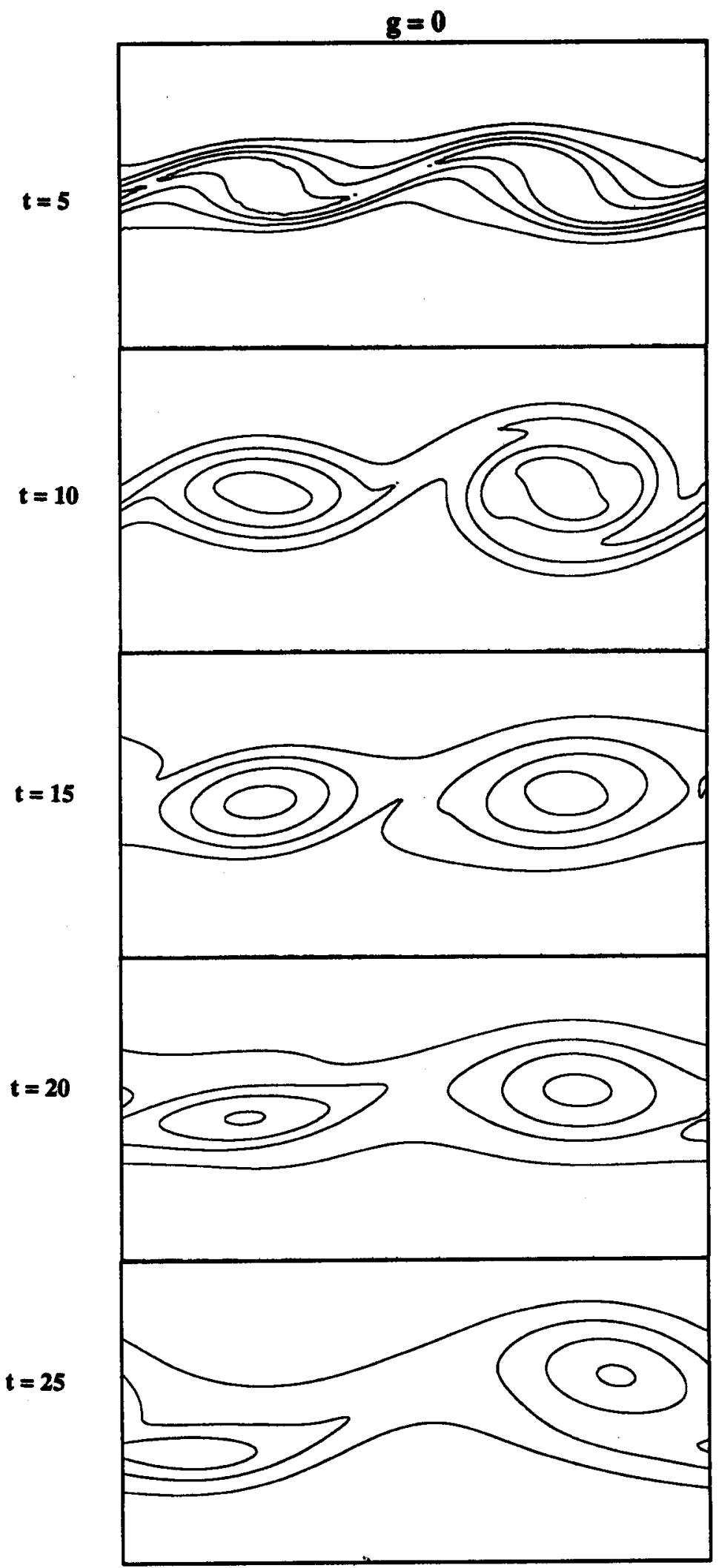

Figure 3. Evolution of $\zeta$ contours at $t=5,10,15,20$ and 25 for case $C$ (single phase). 


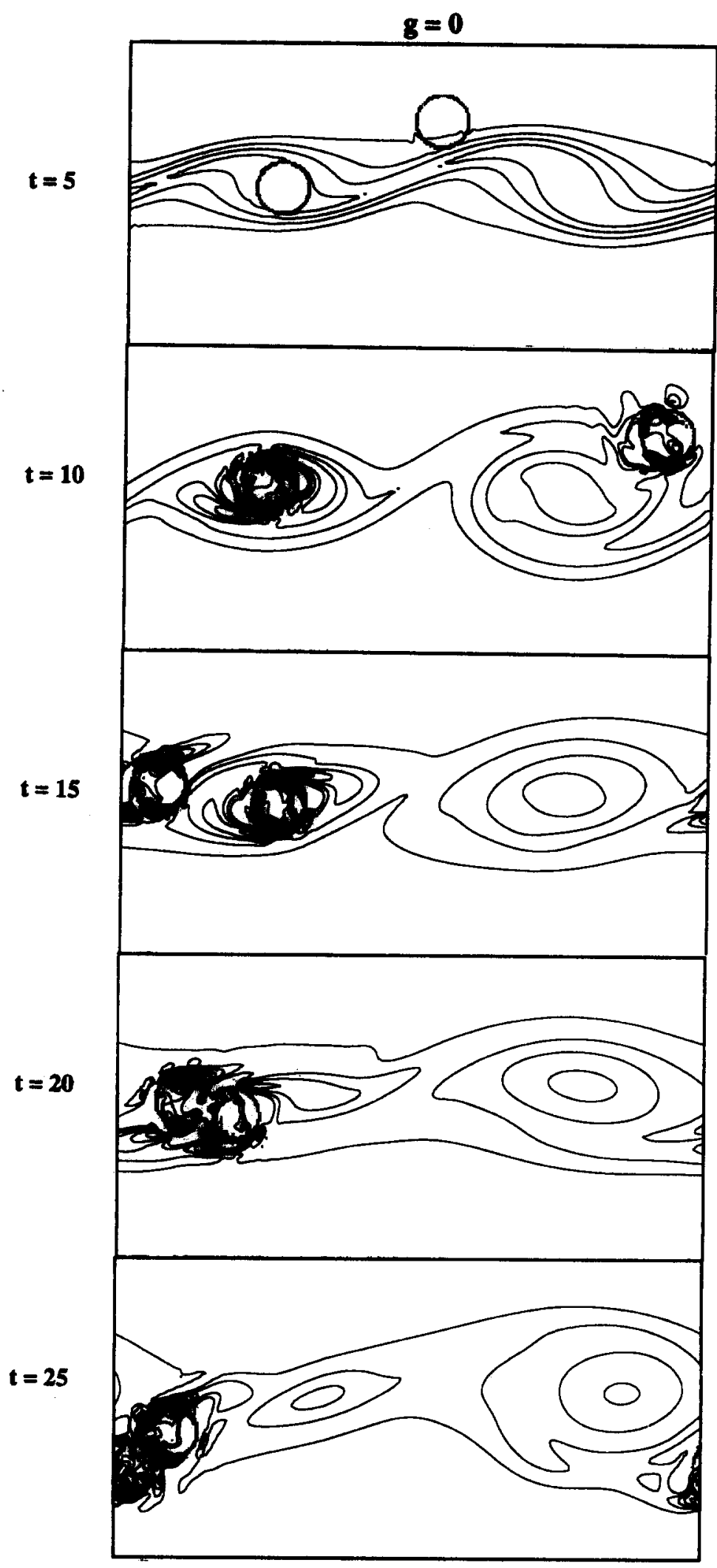

Figure 4. Evolution of $\zeta$ contours at $t=5,10,15,20$ and 25 for case $\mathrm{D}$ (both bubbles with $\rho_{\mathrm{B}}=\rho_{\mathrm{L}}$ and $\boldsymbol{g}=\mathbf{0})$. 


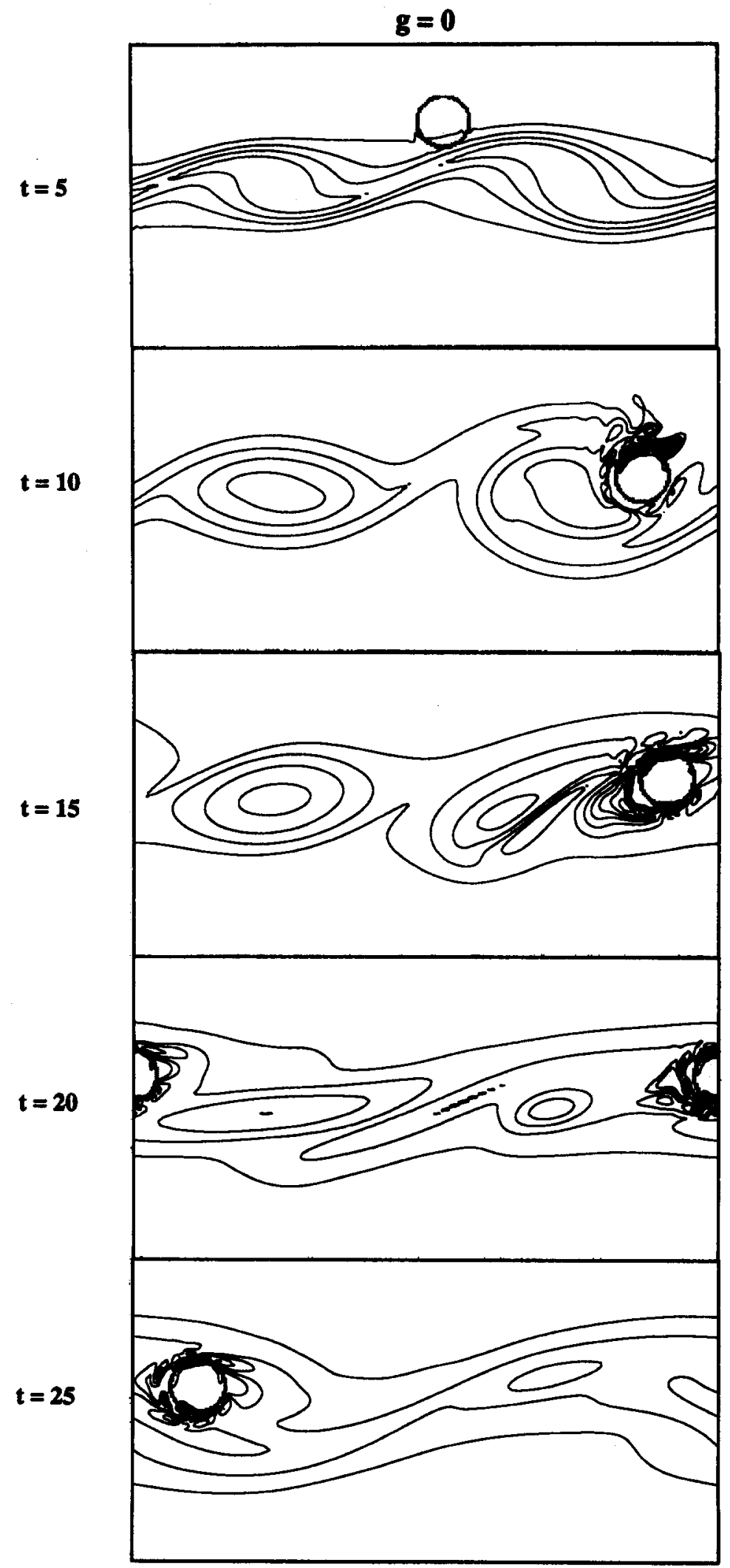

Figure 5. Evolution of $\zeta$ contours at $t=5,10,15,20$ and 25 for case $\mathrm{E}$ (braid bubble with $\rho_{\mathrm{g}} \ll \rho_{\mathrm{L}}$ and $\boldsymbol{g}=\mathbf{0})$. 


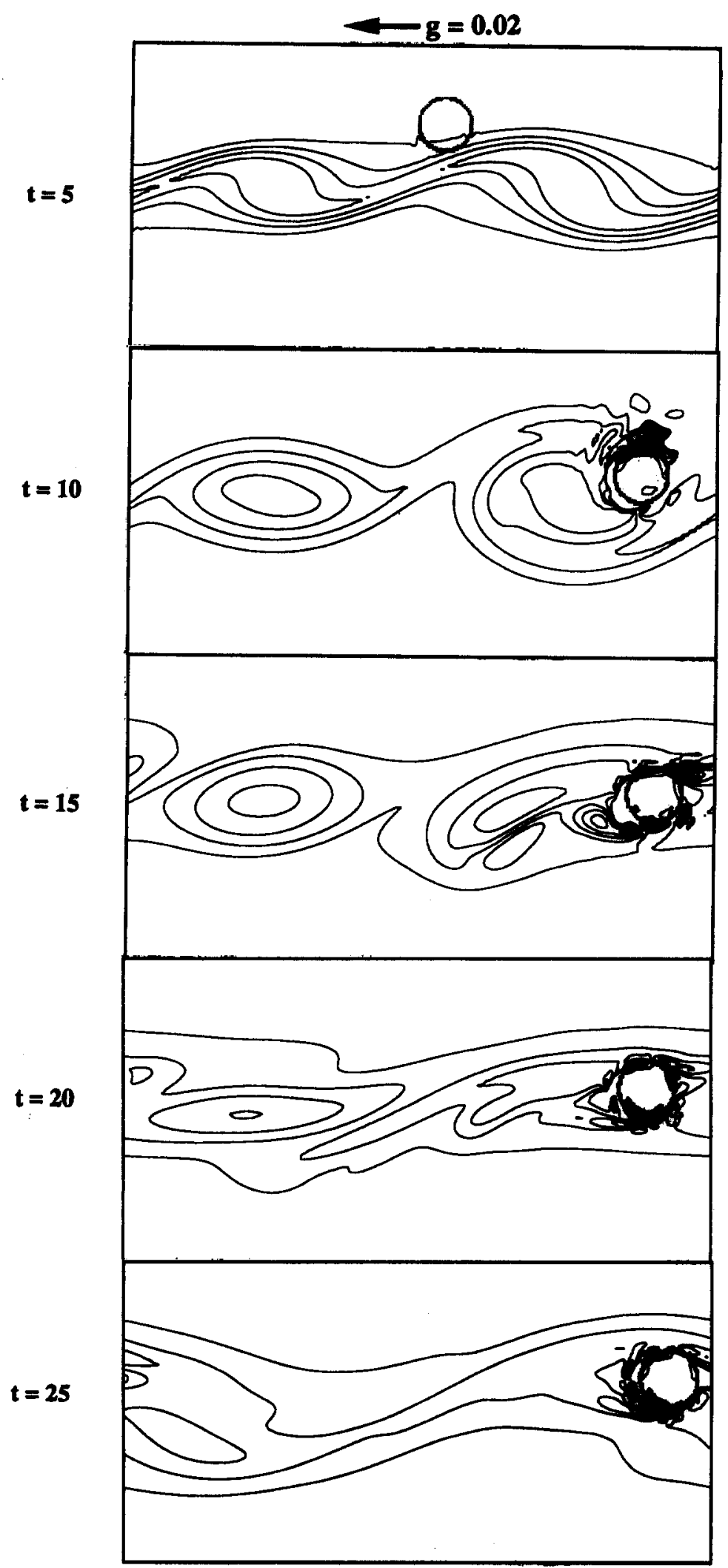

Figure 6.Evolution of $\zeta$ contours at $t=5,10,15,20$ and 25 for case $F$ (braid bubble with $\rho_{\mathrm{B}} \ll \rho_{\mathrm{L}}$ and $g=0.2$ ). 


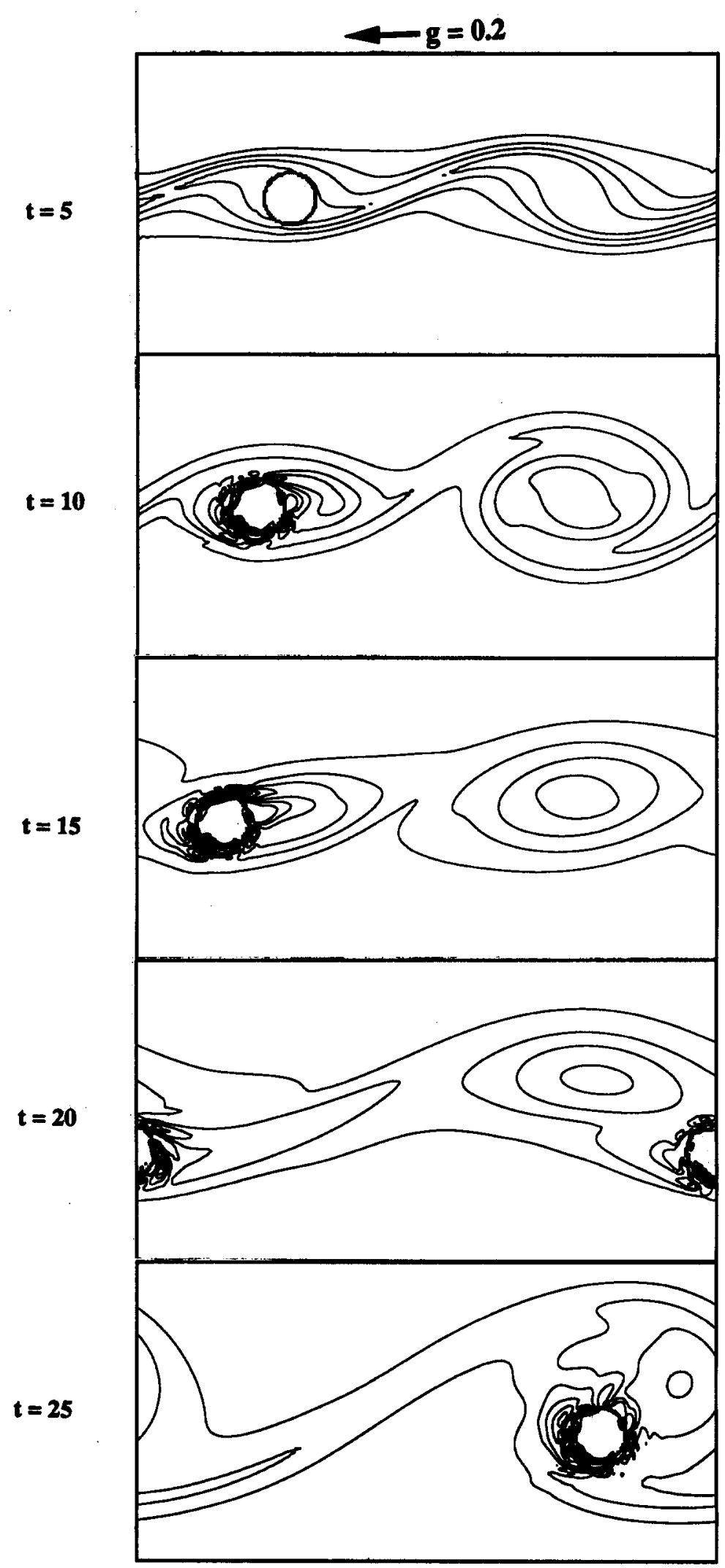

Figure 7. Evolution of $\zeta$ contours at $t=5,10,15,20$ and 25 for case $\mathrm{G}$ (core bubble with $\rho_{\mathrm{B}} \ll \rho_{\mathrm{L}}$ and $g=0.2$ ). 


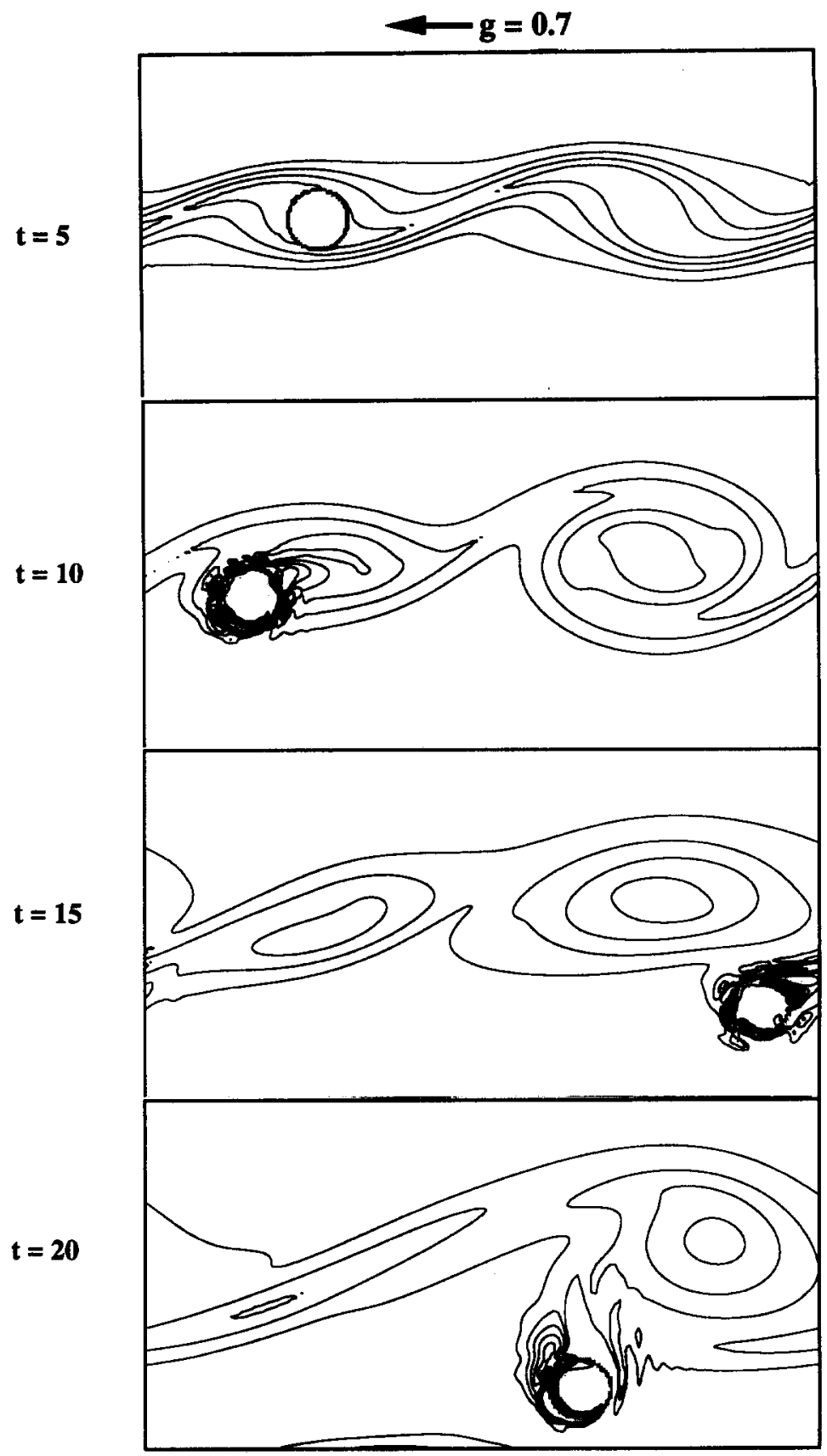

Figure 8. Evolution of $\zeta$ contours at $t=5,10,15$, and 20 for case $\mathrm{H}$ (core bubble with $\rho_{\mathrm{B}} \ll \rho_{\mathrm{L}}$ and $g=0.7$ ).

to a new braid. These results agree well with the similar evolutions obtained by Metcalfe et al. (1978b), for a $\operatorname{Re}_{\delta 0}$ of 332 .

The results related to the modulation of the free shear layer structure by a bubble are discussed next, based on the contours of $\zeta$ for cases D-H (figures 4-8). Figure 4 shows the contours of $\zeta$ for case $D$, for which only surface tension has been introduced and thus includes a displacement effect (due to the added mass), but no centripetal or buoyancy effects. The initial introduction of the bubbles at $t=5$ for this case is shown here, where the bubbles are located at two initial positions, in the left core and above the center braid. While some local effects on the liquid are noted due to the right bubble convection, there are no macroscopic effects on the right eddy or the rest of the flow. By $t=20$, there is not much change in bubble position in the left eddy, whereas 


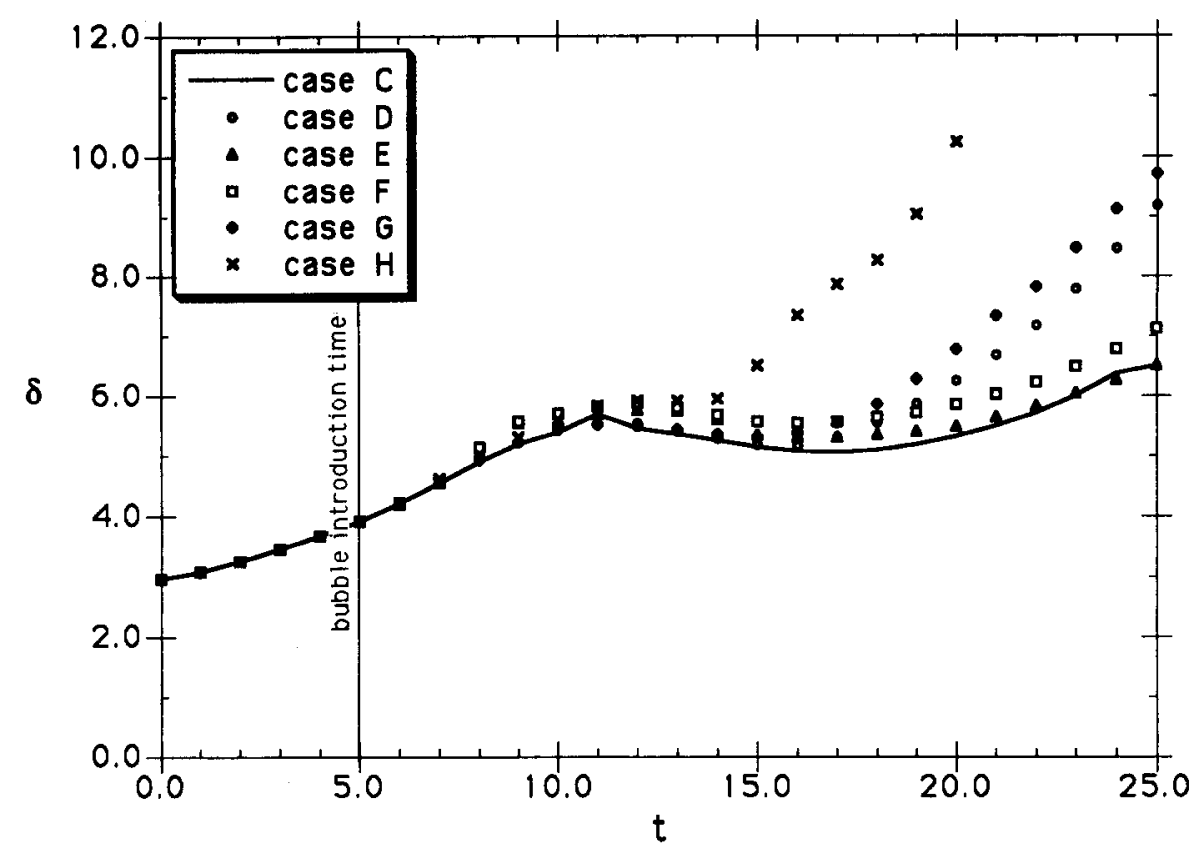

Figure 9. Temporal evolution of shear layer thickness, $\delta$, based on velocity profile.

the right eddy has advected the other bubble through the periodic domain towards the left eddy, after which the bubbles collide but no rupture is allowed numerically. Even at $t=25$, there is little overall change in the eddy structures as compared to the single-phase case other than local bubble wake disturbances. Thus, the displacement effect on flow modulation by itself is apparently small, as expected.

The $\zeta$ contours for case $\mathrm{E}$ are shown in figure 5, where the displacement and centrifugal effects are both present but gravity effect is absent, e.g. $\mathrm{Fe}_{0} \sim \infty$ (see table 1), and where the bubble is introduced above the center braid. By $t=10$, the right bubble is convected similar to case $\mathrm{D}$, except that it is now pulled towards the eddy core (low pressure zone) due to the centripetal effect. Also, by this time it is moving upwards to make the first loop of its trajectory. By $t=15$, the $\zeta$ contours of case $\mathrm{E}$ show that the bubble is still inside the right eddy and the bubble wake has significantly affected the eddy structure resulting in coherency reduction. At this time, the bubble is making a second loop in its trajectory and is getting ready to completely escape the right eddy. By $t=20$, a significant amount of modulation caused by the bubble wake and the eddy crossing in the form of a reduction of coherency of the right eddy, increased stretching and slight pairing enhancement is apparent when case $\mathrm{E}$ is compared to the single phase case at this time. Some of these effects are even shown in the left eddy (e.g. stretching). By $t=25$, the strong dissipation of the right eddy has caused the left eddy (vs the right eddy) to dominate the pairing process.

Figure 6 shows the contours of $\zeta$ for case $F$, in which buoyancy effect has been included by adding gravity $\left(\mathrm{Fe}_{0} \sim 54\right)$, resulting in a theoretical bubble terminal velocity of about 0.21 . The bubble is initiated as in the previous case, but buoyancy retards the bubble at $t=10$ from moving as quickly toward the right eddy core. The flow structures of cases $\mathrm{E}$ and $\mathrm{F}$ at $t=20$ are similar, except that in case $F$, the bubble trajectory crossed through the vortex core and resulted in a stronger core capture, which extended the two-phase momentum transfer time and thus affected the eddy to a greater extent, resulting in additional right eddy dissipation and left eddy domination.

Figure 7 shows the contours of $\zeta$ for case G, which is the same as case F, except that the bubble is now located in the left eddy and the gravity is increased such that $\mathrm{Fe}_{0} \sim 5$ and $V_{1}$ is 0.84 . The increase in $g$ was necessary to allow the bubble to overcome centrifuge and escape the vortex core. The bubble of this case has still stayed close to its initial position until buoyancy finally displaces it to the right, only for the bubble to be then swept downward and to the left by convection forces. 
Note, that in this case the drag force is greater than the buoyancy, such that the convection-buoyancy ratio, $\xi \equiv\left(\Delta u / V_{1}\right)^{2}$, is about 20 . This escape by crossing the eddy results in increased left eddy dissipation resulting in a considerable enhancement of the pairing process.

Figure 8 shows the contours of $\zeta$ for case $\mathrm{H}$, which is the same as case $\mathrm{G}$, except $\mathrm{Fe}_{0} \sim 1.5$, resulting in a bubble terminal velocity of about 1.7 and a $\xi$ of only 5 . The increased buoyancy effect has resulted in the more rapid escape which apparently leads to a lesser reduction of the left eddy coherency than the previous case. This is attributed to a reduced amount of eddy interaction time for modulation to occur, despite the increased perturbation force! Since the bubble moves close to the lower boundary, the calculations were stopped at $t=20$ for this case.

\subsection{Shear layer modulation statistics}

The time history of shear layer thickness for both single and two-phase flows (cases $\mathrm{C}-\mathrm{H}$ ) is plotted in figure 9. The transverse distributions of $\tilde{u}(y)$, not shown here, exhibited profile shapes for all cases at all times which were similar to similarity distributions noted at higher Reynolds numbers (Monkewitz \& Huerre 1982). While the previous section has already discussed the temporal growth of $\delta$, it is worth noting that for case $\mathbf{D}$ (displacement effect only) there is a considerable increase in shear layer thickness with respect to the single-phase case (C) for $t>15$ (figure 9). This increase can be attributed to pseudo-turbulence (caused by the wakes of the two bubbles) by examining the $\zeta$ contours of figure 4 . On the other hand, despite flow modulation the shear layer thicknesses of cases $\mathrm{E}$ (displacement and centripetal effects; right bubble) and $\mathrm{F}$ (all effects, low gravity; right bubble) remain close to that of case C. This is because the right eddy size decrease is balanced by the left eddy size increase. However, the considerable increase in $\delta$ of case $\mathrm{G}$ (all effects; intermediate gravity; left bubble) is due to the earlier pairing enhancement noted previously, and thus may also be considered true flow modulation. Finally, case $\mathrm{H}$ (all effects; high gravity; left bubble) seems to have the highest shear layer thickness, but part of this increase is due to the wake of the bubble (pseudo-turbulence). In general, whenever the coherency of the left eddy is reduced, the overall $\delta$ is increased since the right eddy dominates to a greater extent than usual. However, $\delta$ is on the average unchanged in the cases in which the right eddy has been distorted since the smaller and less effective left eddy is forced to dominate. Experiments by Cebrzynski (1993) have shown that large spherical bubbles can dramatically affect both the eddy coherency and shear layer growth rate, thus supporting the present observations.

To quantify some of the effects noted in previous figures and to study the modifications of the overall fluctuation energies, time histories of the total flow field Favre-averaged parameters $u_{\mathrm{RMS}}^{\prime}(y), v_{\mathrm{RMS}}^{\prime}(y)$, and $\widetilde{u^{\prime} v^{\prime}}(y)$ provide total fluctuation quantities and have been plotted in figures 10-12. There are only small differences between the total $u_{\mathrm{RMS}}^{\prime}$ of case D (displacement effect only; left and right bubbles) and the single-phase case (case $\mathrm{C}$ ), because there is no true flow modulation. Cases $E$ (displacement and centripetal effects; right bubble) and F (all effects, low gravity; right bubble) appear to have initially increased fluctuation levels, due to the wake effects, followed by a significant decrease, presumably due to the lack of eddy coherency. The total $u_{\text {RMS }}^{\prime}$ has almost doubled at later times in case $\mathrm{G}$ (all effects; intermediate gravity; left bubble) in which the right eddy has more coherency and is clearly dominant. The increased total $u_{\text {RMs }}^{\prime}$ for case $G$ over case $\mathrm{H}$ is consistent with the flow modulation noted in figures 7 and 8 . The development of total transverse fluctuation ( $v_{\text {RMS }}^{\prime}$ ) is shown in figure 11 . While the differences between cases are similar to the behaviors for the total $u_{\mathrm{RMS}}^{\prime}$ evolution; the similarity between cases $\mathrm{C}$ and D is especially strong.

Total $\widetilde{u^{\prime} v^{\prime}}$ levels are shown in figure 12 . For case $\mathrm{C}$, this quantity first increases due to the eddy formation and increased coherency. Then, due to the stretching of the eddy structures mentioned earlier, this transverse momentum transport statistic starts to decrease at $t \sim 7$ and reaches a minimum at about $t \sim 13$, for which there is a slight reverse transport due to decreased separation of the two opposing flow regions. Finally, it starts increasing again at $t \sim 13$ due to pairing, which results in increased coherency. Variations of the bubbly flow cases from the baseline case (C) seems to exhibit the same trend followed by total $u_{\mathrm{R} M S}^{\prime}$ and $v_{\mathrm{RMS}}^{\prime}$ although with a negative phase shift in time. The mechanisms cited previously are thus consistent with the results observed. One may also note that the pseudo-turbulence effects do not show up as significantly for $\widetilde{u^{\prime} v}{ }^{\prime}$ levels, e.g. case $\mathrm{H}$. 


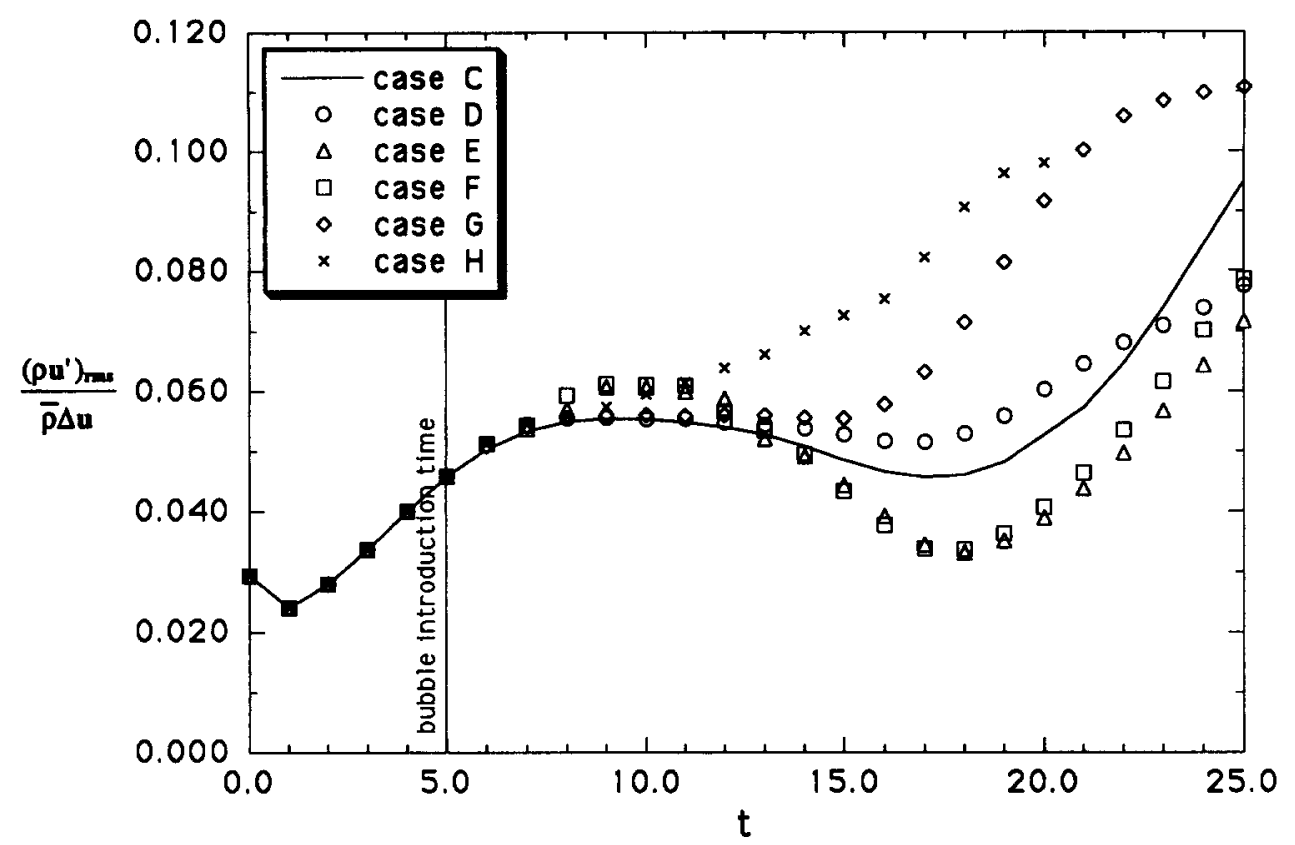

Figure 10. Total Favre-averaged $u_{\mathrm{RMS}}^{\prime}$ vs time for cases $\mathrm{C}$ (single-phase), D (both bubbles with $\rho_{\mathrm{B}}=\rho_{\mathrm{L}}$ and $g=0$ ), $E$ (braid bubble with $\rho_{\mathrm{B}} \ll \rho_{\mathrm{L}}$ and $\boldsymbol{g}=0$ ), F (braid bubble with $\rho_{\mathrm{B}} \ll \rho_{\mathrm{L}}$ and $\boldsymbol{g}=0.2$ ), G (core bubble with $\rho_{\mathrm{B}} \ll \rho_{\mathrm{L}}$ and $g=0.2$ ) and $\mathrm{H}$ (core bubble with $\rho_{\mathrm{B}} \ll \rho_{\mathrm{L}}$ and $g=0.7$ ).

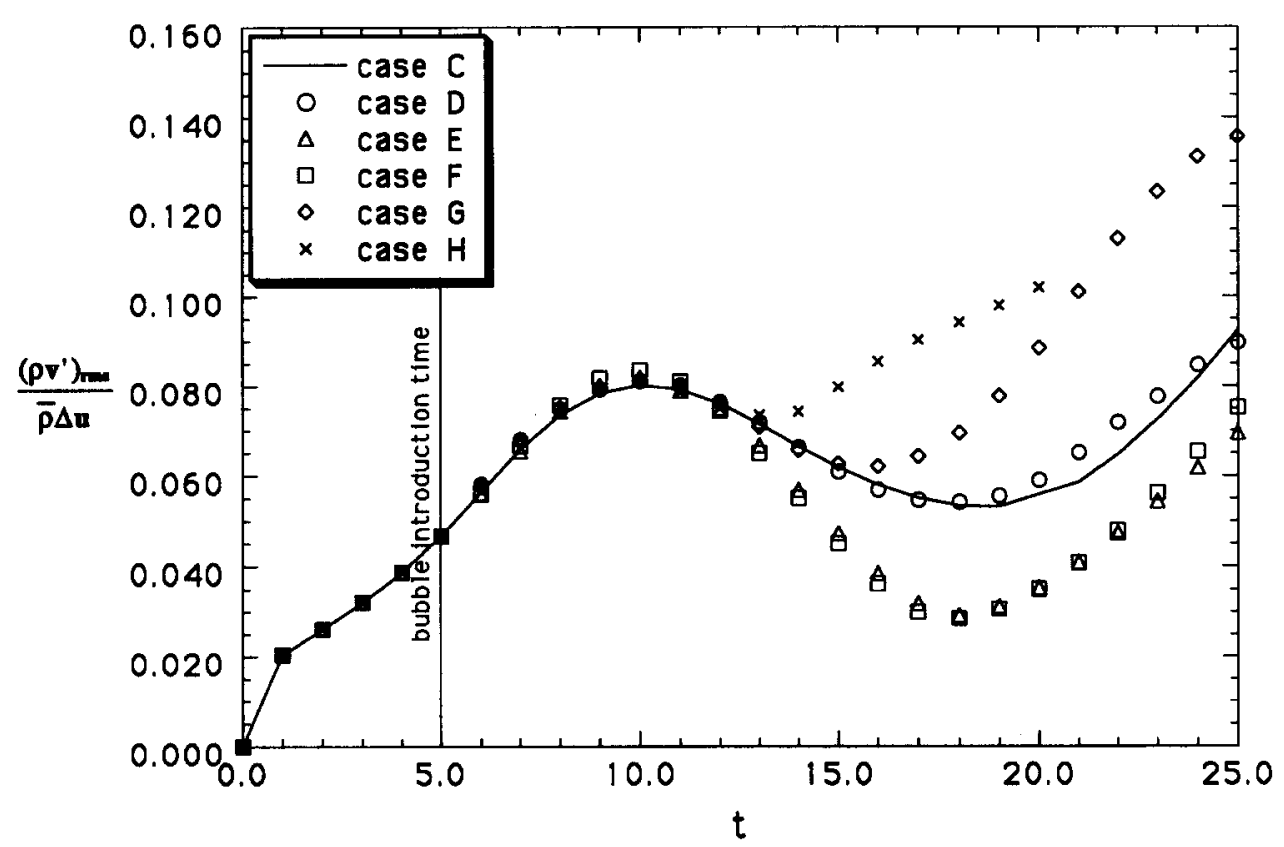

Figure 11. Total Favre-averaged $v_{\mathrm{RMS}}^{\prime}$ vs time for cases $\mathrm{C}$ (single-phase), D (both bubbles with $\rho_{\mathrm{B}}=\rho_{\mathrm{L}}$ and $g=0$ ), E (braid bubble with $\rho_{\mathrm{B}} \ll \rho_{\mathrm{L}}$ and $g=0$ ), F (braid bubble with $\rho_{\mathrm{B}} \ll \rho_{\mathrm{L}}$ and $g=0.2$ ), G (core bubble with $\rho_{\mathrm{B}} \ll \rho_{\mathrm{L}}$ and $g=0.2$ ) and $\mathrm{H}$ (core bubble with $\rho_{\mathrm{B}} \ll \rho_{\mathrm{L}}$ and $g=0.7$ ). 


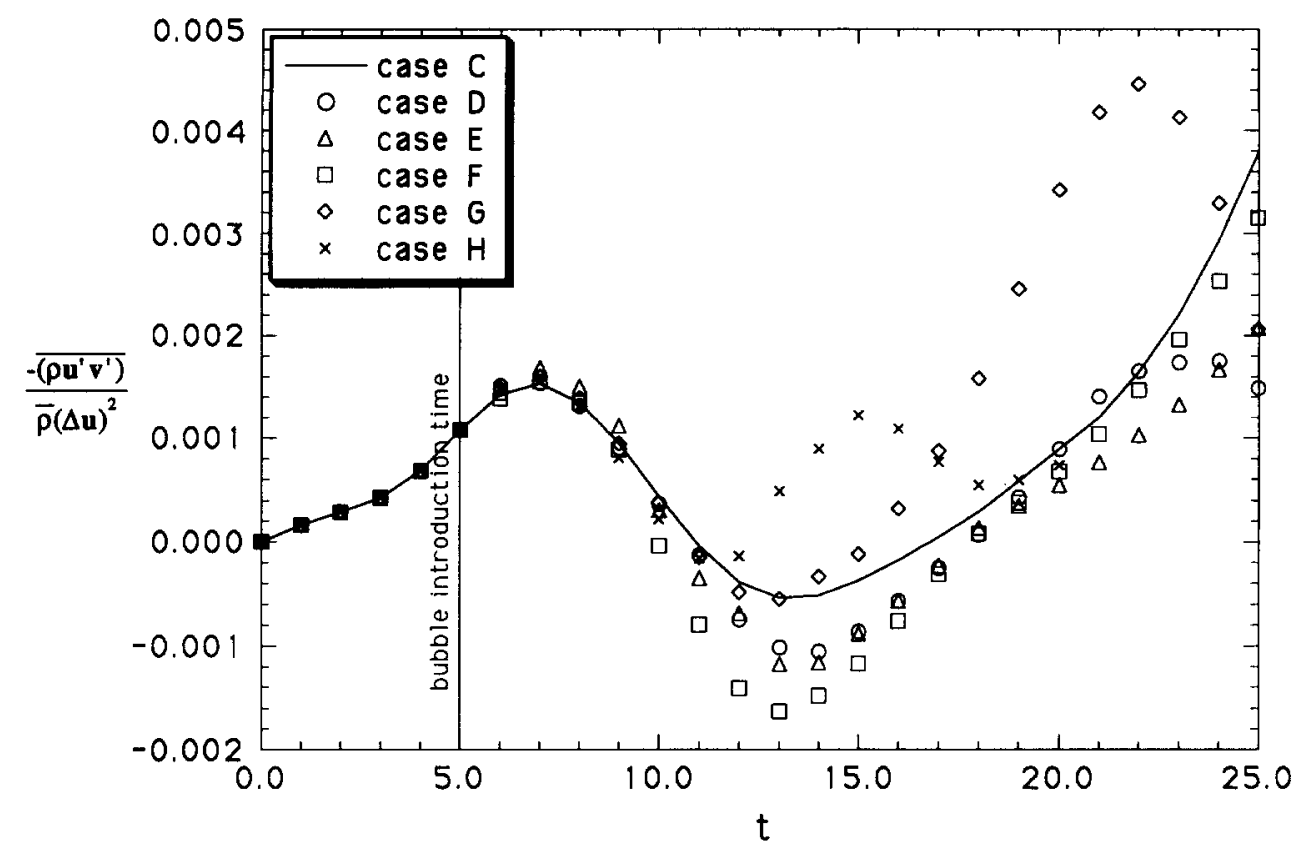

Figure 12. Total Favre-averaged $-\widetilde{u^{\prime} v^{\prime}}$ vs time for cases $\mathrm{C}$ (single-phase), D (both bubbles with $\rho_{\mathbf{B}}=\rho_{\mathbf{L}}$ and $\boldsymbol{g}=0$ ), E (braid bubble with $\rho_{\mathrm{B}} \ll \rho_{\mathrm{L}}$ and $\boldsymbol{g}=0$ ), F (braid bubble with $\rho_{\mathrm{B}} \ll \rho_{\mathrm{L}}$ and $\boldsymbol{g}=\mathbf{0 . 2}$ ), $\mathrm{G}$ (core bubble with $\rho_{\mathrm{B}} \ll \rho_{\mathrm{L}}$ and $\boldsymbol{g}=0.2$ ) and $\mathrm{H}$ (core bubble with $\rho_{\mathrm{B}} \ll \rho_{\mathrm{L}}$ and $\boldsymbol{g}=0.7$ ).

This important discrimination between flow modulation and local bubble induced distortion (pseudo-turbulence) was further supported by examining transverse distributions of $\widetilde{u^{\prime} v}(y)$, not shown here.

\section{CONCLUSIONS}

The temporal evolution of a free shear layer with bubbles has been simulated with the resolved Navier-Stokes equations employed throughout. The bubble size is comparable with the thickness of the free shear layer and we have investigated the effect of introducing the bubbles either into the center of the vortex or just above the braid region. By introducing displacement, density difference and gravity effects one at a time, the importance of each was independently explored. In general, bubbles placed in the vortex core were initially trapped and did not significantly affect the flow until they escaped (and cut through the eddy), similarly bubbles placed above the braid yielded modulation of the eddy only when passing near the vortex core. Buoyancy (even with small gravity values) and centripetal effects were responsible for such interactions, whereas the displacement effect alone did not modify the flow field in a significant macroscopic manner. Modulation typically resulted in reduced eddy coherency and size when an eddy crossing occurred, and appears to be primarily correlated to the time duration of the bubble crossing an eddy, related to eddy Froude number and the convection-buoyancy ratio. However, the enhancement of pairing and the unsteady bubble wake effect (pseudo-turbulence) can both lead to a net increase in fluctuation levels. For an LES subgrid model, this indicates that bubbles can both dissipate eddies of their size, while allowing perturbations that may yield increased energy at larger scales if eddy pairing mechanisms are present, either of which would modify the turbulence spectrum. For such modeling, it is important to discriminate between true vortex flow modulation and bubble wake perturbations (pseudo-turbulence). This was illustrated by comparison of the fluctuation statistics and the flow field visualization.

Acknowledgements - This work was supported by the Office of Naval Research (ONR) under contracts N00014-92-J-1157 and N00014-91-J-1084 with Dr Edwin Rood as technical monitor. The National Center for Supercomputing Applications (NCSA) at the University of Illinois at Urbana-Champaign (UIUC), under 
grant number CBT920031N, furnished the Cray computer time used for the numerical calculations. We wish to acknowledge the help of Mr James Lin and Sean Freeburger, B.S. graduates of the Aeronautical and Astronautical engineering department at UIUC, for their work in flow visualization and data reduction.

\section{REFERENCES}

BRown, G. L. \& RoshKo, A. 1974 On density effects and large structure in turbulent mixing layers. J. Fluid Mech. 64, 775-816.

CEBRzYNSKI, M. S. 1993 Turbulence modulation of a bubbly free shear layer. M.S. Thesis, Department of Aeronautical and Astronautical Engineering, University of Illinois at UrbanaChampaign.

Chahine, G. L. \& Duraiswami, R. 1992 Dynamical interactions in a multi-bubble cloud. J. Fluids Engng. 114, 680-686.

Collins, R. 1965 Structure and behavior of wakes behind two-dimensional air bubbles in water. Chem. Engng Sci. 20, 851-853.

DAvIs, F. 1993 Visualization of turbulence modulation with large particles. M.S. Thesis, Department of Aeronautical and Astronautical Engineering, University of Illinois at UrbanaChampaign.

Faeth, G. M. 1987 Mixing, transport and combustion in sprays. Prog. Energy Combust. Sci. 13, 293-345.

Gore, R. A. \& Crowe, C. T. 1989 Effect of particle size on modulating turbulence intensity. Int. J. Multiphase Flow 15, 279-285.

Ho, C.-M. \& HuANG, L. S. 1982 Subharmonics and vortex merging in a mixing layer. J. Fluid Mech. 119, 443-473.

Lopez de Bertodano, M., Lahey, R. T. \& Jones, O. C. 1992 Development of a $k-c$ model for bubbly two-phase flow. Center for Multiphase Research, Rensselaer Polytechnic Institute.

MetCalfe, R. W., Hussain, A. K. M. F., Menon, S., \& Hayakawa, M. 1987a Coherent structures in a turbulent mixing layer: a comparison between direct numerical simulations and experiments. Turbulent Shear Flows Proc. 5, 110-123.

Metcalfe, R. W., Orszag S. A., Brachet, M. E., Menon, S. \& Riley, J. J. 1987b Secondary instability of a temporally growing mixing layer. J. Fluid Mech. 184, 207-243.

Monkewitz, P. A. \& Huerre, P. 1982 Influence of the velocity ratio on the spatial instability of mixing layers. Phys. Fluids 25, 1137-1143.

Peskin, C. S. 1977 Numerical analysis of blood flow in the heart. J. Comput. Phys. 25, 220-252.

Peyret, R. \& TAYlor, T. D. 1986 Computational Methods for Fluid Flow. Springer, New York.

RiLeY, J. J. \& METCALFE, R. W. 1980 Direct numerical simulation of a perturbed mixing layer. AIAA-80-0274.

RysKin, G. \& LEAL, L. G. 1984 Numerical solution of free boundary problems in fluid mechanics. Part 2. Buoyancy-driven motion of a gas bubble through a quiescent liquid. J. Fluid Mech. 148, 19-35.

SANDham, N. D. \& ReYnolds, W. C. 1989 Some inlet plane effects on the numerically simulated spatially-developing mixing layer. Turbulent Shear Flows 6, 441-454.

SHANKAR, P. N. 1992 On the shape of a two-dimensional bubble in uniform motion. J. Fluid Mech. 244, 187-200.

TaEibi-Rahni, M., Loth, E. \& Tryggvason, G. 1992 Numerical study of turbulence modulation in a bubbly mixing layer. 13th Symposium on Turbulence, University of Missouri-Rolla, B-21.

Tishkoff, J. 1989 Interaction between droplets and gas-phase turbulent flows. Proceedings of the ASCE/ASME Fluids Engineering Division, La Jolla, CA.

UnVERdi, S. O. \& Tryggvason, G. 1992a A front-tracking method for viscous, incompressible, multi-fluid flows. J. Comput. Phys. 100, 25-37.

Unverdi, S. O. \& Tryggvason, G. 1992b Computations of multi-fluid flows. Physica D 60, 70-83.

VANDEN-BROCK, J. M. 1992 Rising bubble in a two-dimensional tube: asymptotic behavior for small values of the surface tension. Phys. Fluids A 4, 2332-2334.

WinaNT, C. D. \& BRowAND, F. K. 1974 Vortex pairing: the mechanism of turbulent mixing layer growth at moderate Reynolds number. J. Fluid Mech. 63, 237-255. 
YuAN, Z. \& Michaelides, E. E. 1992 Turbulence modulation in particulate flows- a theoretical approach. Int. J. Multiphase Flow 18, 779-785.

Zun, I., KLJENAK, I., \& MOZE, S. 1993 Space-time evolution of the non-homogeneous bubble dispersion in upward flow. Int. J. Multiphase Flow 19, 151-172. 